\title{
Microscopic peritoneal carcinomatosis in gastric cancer: Prevalence, prognosis and predictive factors
}

\author{
ENRICO MARIA PASQUAL ${ }^{1}$, SERENA BERTOZZI ${ }^{1}$, AMBROGIO P. LONDERO ${ }^{2}$, \\ DENISE BRANDOLIN $^{1}$, LAURA MARIUZZI ${ }^{1}$, ALESSANDRO DE PELLEGRIN ${ }^{3}$, \\ STEFANO BACCHETTI $^{1}$, LORIS ZORATTI ${ }^{4}$, ROBERTO PETRI ${ }^{5}$, CARLO DELLA BIANCA ${ }^{6}$, \\ DANIELE SNIDERO $^{7}$, GIOVANNI TERROSU ${ }^{1}$, ALESSANDRO UZZAU ${ }^{1}$, ANDREA RISALITI ${ }^{1}$, \\ CARLA DI LORETO $^{1}$, STEFANO PIZZOLITTO $^{3}$, MAURIZIO ZILLI ${ }^{4}$ and GIOVANNI DE MANZONI ${ }^{8}$

\footnotetext{
${ }^{1}$ Department of Medical Area, University of Udine, Santa Maria della Misericordia University Hospital Udine, I-33100 Udine;

${ }^{2}$ Department of Obstetrics and Gynaecology, Monfalcone Hospital, I-34074 Monfalcone; Departments of ${ }^{3}$ Pathology,

${ }^{4}$ Gastroenterology and ${ }^{5}$ Surgery, Santa Maria della Misericordia University Hospital Udine, I-33100 Udine;

${ }^{6}$ Department of Surgery, Tolmezzo Hospital, I-33028 Tolmezzo; ${ }^{7}$ Department of Surgery, San Daniele Hospital, I-33038 San Daniele;

${ }^{8}$ Department of Upper Gastrointestinal Surgery, The Civil Hospital of Verona University, I-37126 Verona, Italy
}

Received August 10, 2015; Accepted February 23, 2017

DOI: $10.3892 / \mathrm{ol} .2017 .7442$

\begin{abstract}
Peritoneal carcinomatosis (PC) is typically identified in advanced stage gastric cancer and is frequently considered to be an incurable disease. Along with macroscopic PC, microscopic PC may be diagnosed through pathological examination of tissue specimens and is not detectable during surgical intervention. The present study aimed to analyse the prevalence, prognostic value and predictive factors for microscopic PC. In the present retrospective study, data from patients with epithelial gastric cancer that were treated with curative intent surgery were examined. Patients with macroscopic PC were excluded. Additionally, the study population was divided into two groups based on the presence or absence of microscopic PC. The prevalence of microscopic PC was $5.5 \%$. Microscopic PC exhibited a significant negative effect on overall survival. In addition, multivariate analyses revealed
\end{abstract}

Correspondence to: Professor Enrico Maria Pasqual, Department of Medical Area, University of Udine, Santa Maria della Misericordia University Hospital Udine, 15 Piazzale Santa Maria della Misericordia, I-33100 Udine, Italy

E-mail: enricomaria.pasqual@uniud.it

Dr Ambrogio P. Londero, Department of Obstetrics and Gynaecology, Monfalcone Hospital, 1 Galvani, I-34074 Monfalcone, Italy

E-mail: ambrogio.londero@gmail.com

Abbreviations: AUC, area under the curve; CI, confidence interval; CRS, cytoreductive surgery; HIPEC, hyperthermic intraperitoneal chemotherapy; HR, hazard ratio; IQR, inter-quartile range; LVI, lymphatic and vascular invasion; OS, overall survival; PC, peritoneal carcinomatosis; ROC, receiver operator characteristic; TNM, tumor-node-metastasis

Key words: gastric cancer, epithelial gastric tumour, OS, PC, microscopic PC, predictive factors that the significant predictive factors for the presence of microscopic PC were adenocarcinoma of a diffuse type, lymphatic and vascular invasion, cancer location at the site of previous gastric surgery and a tumour extent $>\mathrm{T} 2$. In particular, the presence of lymphatic and vascular invasion was the most significant predictive factor. These results indicate that $\geq 5.5 \%$ of patients with gastric cancer who undergo surgery with a curative intent may benefit from more aggressive loco-regional treatment against microscopic $\mathrm{PC}$ at the time of surgery.

\section{Introduction}

Although the incidence of gastric cancer has decreased, it remains one of the most common types of cancer and one of the leading causes of cancer-associated mortality worldwide, including the region of North-East Italy (1-3). In this region of Italy, the yearly gastric cancer incidence was estimated to be 23.7/100,000 males and 11.7/100,000 females (3).

Peritoneal carcinomatosis (PC) in gastric cancer is considered a distant metastasis and is detected in $\sim 60 \%$ of patients with gastric cancer subsequent to typical curative treatment (4). PC is frequently a fatal disease and has limited treatment options. In particular, the Evolution of Peritoneal Carcinomatosis 1 study demonstrated a median overall survival (OS) of 3.1 months in patients with PC (5). Cytoreductive surgery (CRS) associated with hyperthermic intraperitoneal chemotherapy (HIPEC) has been revealed to exhibit a beneficial effect against PC in gastric cancer in a phase III trial (6).

As PC is difficult to diagnose in the early stages of the disease through clinical and instrumental techniques $(7,8)$, it is important to quantify the prevalence of microscopic PC at the time of primary surgery, and to analyse the possible risk factors for the presence of PC. Additionally, it may be useful to enable the planning of extensive loco-regional treatments, and to potentially perform CRS with HIPEC in selected patients 
who possess a high probability of exhibiting microscopic PC at the time of primary surgery.

The aim of the present study was to analyse the prevalence of microscopic PC in patients receiving surgical treatment for epithelial gastric tumours, examine the prognostic value of microscopic PC through OS analysis and identify potential predictive factors for the presence of microscopic PC.

\section{Materials and methods}

Patients. The present study was retrospective and included 399 patients whom received surgery for epithelial gastric tumours between January 2001 and July 2013 and the pathological specimen was assessed at the University Hospital of Udine (Udine, Italy). Follow-up data was available until July 2014. The present study was approved by the internal review board of the Department of medical area (University of Udine, Udine, Italy) and was conducted in accordance with the Helsinki Declaration and following the dictates of the general authorisation to process personal data for scientific research purposes by the Italian Data Protection Authority.

Only patients that had undergone an adequate gastric resection, with negative microscopic margins and adequate resection of regional lymphatics, including greater and lesser omenta were included in the present study. All patients that presented with macroscopic PC at the time of surgery were excluded from the analyses, even if they underwent radical surgery with complete resection of peritoneal metastases.

A total of 403 patients with epithelial gastric cancer that underwent complete surgical resection, including greater and lesser omenta, were identified. Among these, 399 patients were eligible for the present study. A total of 4 patients with macroscopic PC were excluded from the analyses. The clinicopathological characteristics of the patients included in the present study are presented in Table I.

Patient data. Data for the selection of patients were gathered from University Hospital of Udine clinical and pathological files. The primary outcomes evaluated in the present study were the prevalence of microscopic $\mathrm{PC}$ and the OS rate. The analyses of predictive factors for the presence of microscopic PC was considered as a secondary outcome. The following data about patient and tumour characteristics were collected: Patient age (at diagnosis), sex, weight, tobacco usage and alcohol consumption, and the tumour histotype, location, size, lymph node status, grading, lymphatic and vascular invasion (LVI), and whether microscopic PC was present (determined by pathological examination of tissue samples obtained during gastric resection). In addition, the therapeutic management used, including neoadjuvant and adjuvant chemotherapy, and radiotherapy, was analysed. Furthermore, information concerning post-operative follow-up and eventual cancer-associated mortality was collected in all cases from the clinical files of University Hospital of Udine.

Tumour classification and staging. Tumour stage was defined according to the seventh edition of the Tumor-Node-Metastasis (TNM) staging classification for carcinomas of the stomach of the American Joint Committee on Cancer and Union for
International Cancer Control. Tumour histology was classified according to the Lauren classification of gastric tumours (9) and tumour grading was performed according to the fourth edition of the World Health Organization classification of tumours of the digestive system (10). LVI was considered as present or absent, and the presence of LVI was defined as microscopically-detected lymphovascular invasion in $\geq 1-2$ lymphatics or small veins in a mounted specimen containing the deepest portion of the tumour on a glass slide, as previously described (11). Tumour location was divided as follows: Fundus, body, antrum-pylorus or site of previous gastric surgery. In the category 'site of previous gastric surgery', 3 patients who had undergone previous gastric bypass surgery for severe obesity and 2 patients who underwent previous Billroth II gastric resection for peptic ulcer disease were included (in the remaining 5 cases the reason of surgery for benign conditions was unknown).

Treatment. All patients included in the present study were classified as type III according to the Siewert-Stein classification of adenocarcinomas of the esophagogastric junction (12) and were treated according to national comprehensive cancer network guidelines for gastric cancer (12). Routine post-operative follow-up was performed every 3-6 months for the first 2 years, every 6-12 months from 2.5-5 years and every 12 months thereafter. Blood cell counts and chemistry profiling were performed, in addition to diagnostic imaging or endoscopy when appropriate.

Outcomes and groupings. In the present study, OS was defined as the time from the primary surgical procedure to mortality due to gastric cancer or the last registered follow-up. The definition of microscopic PC was as previously defined (13): The presence of peritoneal nodules demonstrated to be of a gastric cancer origin without any microscopic evidence of lymph node architecture or tissue, which were not identified by macroscopic intraoperative examination. The study population was divided into two groups according to the presence or absence of microscopic PC.

Statistical analysis. Data were analysed using R software (version 3.1.0; R Foundation for Statistical Computing, Vienna, Austria), and $\mathrm{P}<0.05$ was considered to indicate a statistically significant difference. Univariate analysis was performed using Fisher's exact test or $\chi^{2}$ test in the case of categorical variables, and a Wilcoxon signed-rank test or unpaired t-test in the case of continuous variables. In addition, Kaplan-Meier survival curves were constructed, and a log-rank test was used to compare the OS rates between the study groups. In addition, the Cox proportional hazard regression model was used to assess OS and adjusted in the multivariate analysis for possible confounding factors. Univariate and multivariate logistic regression analyses were performed with the presence of microscopic PC considered a dependent variable, and with the possible predictors selected from the univariate analysis considered as independent variables. The final multivariate model was obtained through stepwise regression analyses and was determined to be the most predictive model for the presence of microscopic PC. Receiver operator characteristic (ROC) curves were 
Table I. Clinicopathological characteristics of the study population.

$\begin{array}{lc}\text { Clinicopathological } & \begin{array}{c}\text { Percentage of patients } \\ \text { characteristic }\end{array} \\ \text { (no./total) }\end{array}$

\section{Sex}

$$
\text { Female }
$$

Male

Tobacco smoke

Alcohol consumption

Tumour characteristics

Histological type

$\begin{array}{lc}\text { Adenocarcinoma intestinal type } & 64.4(257 / 399) \\ \text { Adenocarcinoma diffuse type } & 25.8(103 / 399) \\ \text { Adenocarcinoma mixed type } & 9.8(39 / 399) \\ \text { Location of cancer } & \\ \text { Fundus } & 14.8(59 / 399) \\ \text { Body } & 44.9(179 / 399) \\ \text { Antrum-pylorus } & 37.8(151 / 399) \\ \text { Site of previous gastric surgery } & 2.5(10 / 399) \\ \text { Tumour extent } & \\ \text { Tis } & 1.3(5 / 399) \\ \text { T1 } & 17.5(70 / 399) \\ \text { T2 } & 28.6(114 / 399) \\ \text { T3 } & 45.4(181 / 399) \\ \text { T4 } & 7.3(29 / 399)\end{array}$

Nodal status

N0

$\mathrm{N} 1$

$37.8(151 / 399)$

$30.3(121 / 399)$

$19.5(78 / 399)$

$12.3(49 / 399)$

N3

$39.6(158 / 399)$

$28.8(115 / 399)$

Stage III

Tumour grading

G1

G2

$7.1(28 / 392)$

$33.4(131 / 392)$

G3

$59.4(233 / 392)$

Lymphatic and vascular invasion
$21.6(86 / 399)$ was 67 kilograms [interquartile range (IQR), 55-75 (data not shown)]. The majority of the patients were male $(62.2 \%$; 248/399). The median follow-up time was 25 months (IQR, 11-72; data not shown). Histological analysis revealed that $64.4 \%$ of patients $(257 / 399)$ exhibited the histotype of adenocarcinoma of the intestinal type, $25.8 \%$ of patients $(103 / 399)$ exhibited adenocarcinoma of a diffuse type and $9.8 \%$ of patients (39/399) exhibited adenocarcinoma of a mixed type. Microscopic PC exhibited a prevalence of 5.5\% (22/399; data not shown). Additionally, the majority of patients were TNM stage II and tumour grading G3. Lymphatic and vascular invasion was present in $21.6 \%$ of patients $(86 / 399)$.

Clinicopathological characteristics of patients with epithelial gastric cancer in the absence or presence of microscopic PC. Table II summarises the differences between patients with or without microscopic PC. No significant differences were identified in the patients with or without microscopic PC for age at diagnosis $[70.95$ years $( \pm 13.88)$ vs. 69.92 years $( \pm 11.59)$ $\mathrm{P}>0.05]$. and weight $[61.00( \pm 6.56)$ vs. $67.83( \pm 16.38) \mathrm{P}>0.05]$. (data not shown). Among the patients with microscopic PC, a significantly higher prevalence of: Tobacco smoking (unfortunately with 279 missing data), adenocarcinoma of a diffuse type, T4 tumour extent, N2 nodal status, TNM stage III, tumour grading G3 and lymphatic and vascular invasion. Typically, a higher tumour extent and positive nodal status were associated with the presence of microscopic PC. In addition, tumours arising from previous non-oncological gastric surgery were more common in the group of patients with microscopic PC compared with the patients without microscopic PC, yet no significant difference was observed.

Predictive factors for microscopic PC. Table III summarises the univariate and the multivariate logistic regression analyses, highlighting the most predictive factors considered in the final multivariate model identified by step-wise regression analysis. Tobacco smoke was excluded from this analysis due to missing data. The final multivariate regression model considered the following factors: Adenocarcinoma of a diffuse type, lymphatic and vascular invasion, cancer location at the site of a previous gastric surgery and tumour extent $>\mathrm{T} 2$. This model presented an AUC of the ROC curve of $88.7 \%$ [95\% confidence interval (CI), 80.9-96.5\%]. The most significant predictive factor was the presence of lymphatic and vascular invasion.

OS rate of patients with epithelial gastric cancer in the absence or presence of microscopic $P C$. The differences in $\mathrm{OS}$ between patients with and without microscopic PC were analysed. Fig. 1 demonstrates a significant difference in the OS between the whole population, the T2 tumour extent group and the T3 tumour extent group. Fig. 2 illustrates that there was a significant difference in OS in the TNM stage II and III groups between patients with and without microscopic PC. The 1-year OS rate in patients without microscopic PC was 77.5\% (95\% CI, 73.1-82.2), and in patients with microscopic PC it was $40.1 \%$ (95\% CI, 24.8-67.6\%) (Fig. 1A). The 2-year OS rate was $63.3 \%$ (95\% CI, 58.1-68.9\%) and $12.7 \%(95 \%$ CI, 3.7-43.8\%) in patients without and with microscopic PC,

respectively (Fig. 1A). A Cox proportional hazard regression
Clinicopathological characteristics of patients with epithelial gastric cancer. Table I summarises the clinicopathological characteristics of the study population. The mean

age at diagnosis was $69.97 \pm 11.71$ years and the median weight

produced, and the area under the curve (AUC) was used to assess the accuracy of the prediction of the model.

\section{Results}


Table II. Clinicopathological characteristics of the study population subdivided by the presence or absence of microscopic PC.

Microscopic PC status,

percentage of patients (no./total)

Clinicopathological characteristic

Absent $(n=377)$

Present $(n=22)$

P-value

Sex

Female

Male

Tobacco smoke

Alcohol consumption

Tumour characteristics

Histological type

Adenocarcinoma intestinal type

Adenocarcinoma diffuse type

Adenocarcinoma mixed type

Location of cancer

Fundus

Body

Antrum-pylorus

Site of previous gastric surgery

Tumour extent

Tis

T1

$\mathrm{T} 2$

T3

T4

Nodal status

$\begin{array}{ll}\text { N0 } & 39.3(148 / 377) \\ \text { N1 } & 30.2(114 / 377) \\ \text { N2 } & 18.6(70 / 377) \\ \text { N3 } & 11.9(45 / 377)\end{array}$

TNM stage

Stage 0

Stage I

Stage II

Stage III

Tumour grading

$\begin{array}{ll}\text { G1 } & 7.6(28 / 370) \\ \text { G2 } & 34.9(129 / 370) \\ \text { G3 } & 57.6(213 / 370) \\ \text { Lymphatic and vascular invasion } & 17.8(67 / 377)\end{array}$

$66(249 / 377)$

$24.4(92 / 377)$

$9.5(36 / 377)$

$15.4(58 / 377)$

$44(166 / 377)$

$38.5(145 / 377)$

$2.1(8 / 377)$

$1.3(5 / 377)$

$18.6(70 / 377)$

$29.2(110 / 377)$

$44.8(169 / 377)$

$6.1(23 / 377)$

$1.3(5 / 377)$

$32.1(121 / 377)$

$39(147 / 377)$

$\begin{array}{rlr}37.9(143 / 377) & 36.4(8 / 22) & 0.883 \\ 62.1 \%(234 / 377) & 63.6(14 / 22) & 0.883 \\ 3.4(4 / 117) & 33.3(1 / 3) & <0.050 \\ 4.3(5 / 117) & 0(0 / 3) & 0.715\end{array}$

$36.4(8 / 22)$

$<0.050$

$50(11 / 22)$

$<0.050$

$13.6(3 / 22)$

0.530

$4.5(1 / 22)$

0.164

$59.1(13 / 22)$

0.167

$27.3(6 / 22)$

0.293

$9.1(2 / 22)$

0.100

$0(0 / 22)$

0.587

$0(0 / 22)$

$<0.050$

$18.2(4 / 22)$

0.267

$54.5(12 / 22)$

0.373

$27.3(6 / 22)$

$<0.050$

$13.6(3 / 22)$

$<0.050$

$31.8(7 / 22)$

0.876

$36.4(8 / 22)$

$<0.050$

$18.2(4 / 22)$

0.386

$0(0 / 22)$

0.587

$0(0 / 22)$

$<0.050$

$50(11 / 22)$

0.305

$50(11 / 22)$

$<0.050$

$0(0 / 22)$

0.181

$9.1(2 / 22)$

$<0.050$

$90.9(20 / 22)$

$<0.050$

$86.4(19 / 22)$

$<0.050$

P-values from $\chi^{2}$ or Fisher's exact test as appropriate where binary data was presented as a percentage. PC, peritoneal carcinomatosis; TNM, tumor node metastasis; Tis, carcinoma in situ.

model analysis was also performed, where microscopic PC demonstrated a hazard ratio (HR) of 4.00 (95\% CI, 2.45-6.53, $\mathrm{P}<0.05$; data not shown). Subsequent to adjustment for TNM stage, cancer location, tumour grading, lymphatic and vascular invasion, age, and neoadjuvant and adjuvant therapies in the multivariate analyses, the HR was 2.02 (95\% CI, 1.10-3.71, $\mathrm{P}<0.05$; data not shown).

\section{Discussion}

Among patients who received radical surgery for gastric cancer, microscopic PC exhibited a prevalence of $5.5 \%$ in the absence of any macroscopic PC. Additionally, microscopic PC elicited a significant negative effect on OS, even in patients with T2 gastric cancer. In the study population, the multivariate model 
Table III. Univariate and multivariate logistic regression analyses.

\begin{tabular}{|c|c|c|c|c|}
\hline \multirow[b]{2}{*}{ Independent variable } & \multicolumn{2}{|c|}{ Univariate analysis } & \multicolumn{2}{|c|}{ Multivariate analysis } \\
\hline & OR $(95 \% \mathrm{CI})$ & P-value & OR $(95 \% \mathrm{CI})$ & P-value \\
\hline Adenocarcinoma diffuse type & $3.11(1.3-7.42)$ & $<0.050$ & $2.92(1.08-7.9)$ & $<0.050$ \\
\hline Site of previous gastric surgery & $4.52(0.9-22.72)$ & 0.067 & $4.87(0.69-34.57)$ & 0.113 \\
\hline Tumour extent $>\mathrm{T} 2$ & $4.17(1.39-12.56)$ & $<0.050$ & $2.59(0.79-8.46)$ & 0.116 \\
\hline Nodal status $>\mathrm{N} 0$ & $3.94(1.15-13.57)$ & $<0.050$ & - & - \\
\hline Tumour grading G3 & $7.37(1.7-32)$ & $<0.050$ & - & - \\
\hline Lymphatic and vascular invasion & $28.64(8.24-99.58)$ & $<0.050$ & $22.65(6.4-80.22)$ & $<0.050$ \\
\hline
\end{tabular}

P-value refers to logistic regression analysis. The dependent variable was the presence of microscopic PC. OR, odds ratio; CI, confidence interval.
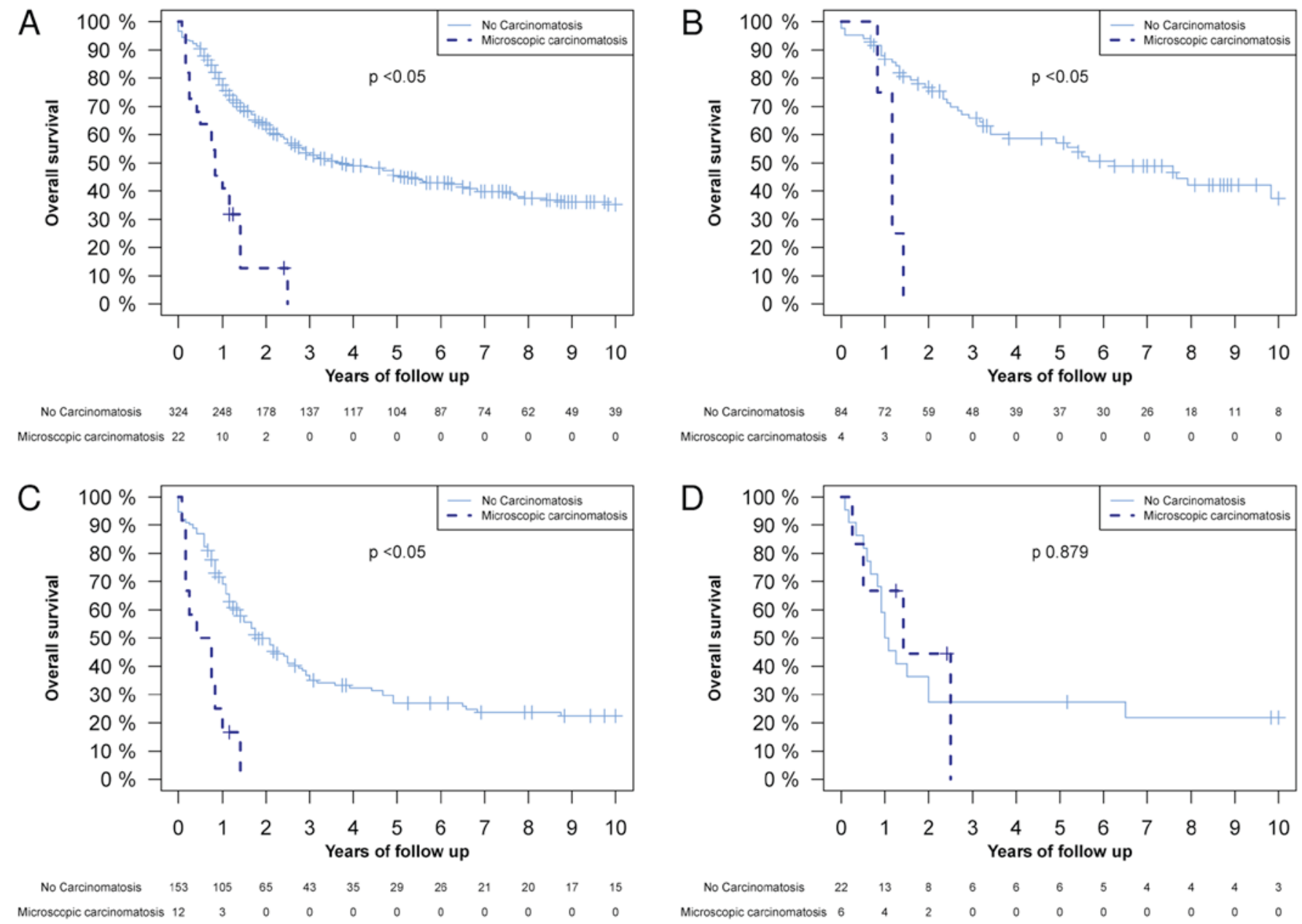

Figure 1. Kaplan-Meier overall survival curves of the following groups, subdivided by the presence or absence of microscopic peritoneal carcinomatosis: (A) The whole study population; (B) T2 tumours; (C) T3 tumours; and (D) of T4 tumours. P-values were calculated using a log-rank test.

demonstrated that adenocarcinoma of a diffuse type, lymphatic and vascular invasion, cancer location at the site of previous gastric surgery and a tumour extent $>\mathrm{T} 2$ were the most predictive factors for microscopic PC, with an AUC of the ROC curve of $88.7 \%$ (95\% CI, 80.9-96.5). In particular, the presence of lymphatic and vascular invasion was revealed to be the most significant and individual predictive factor for microscopic PC.
The primary limitation of the present study was the retrospective design and the limited number of patients affected by microscopic PC. Other possible biases may be the limited information concerning adjuvant and neoadjuvant treatment protocols used for specific patients, and the incomplete information concerning the timings of disease recurrence. Despite the limited number of patients, significant differences in the 

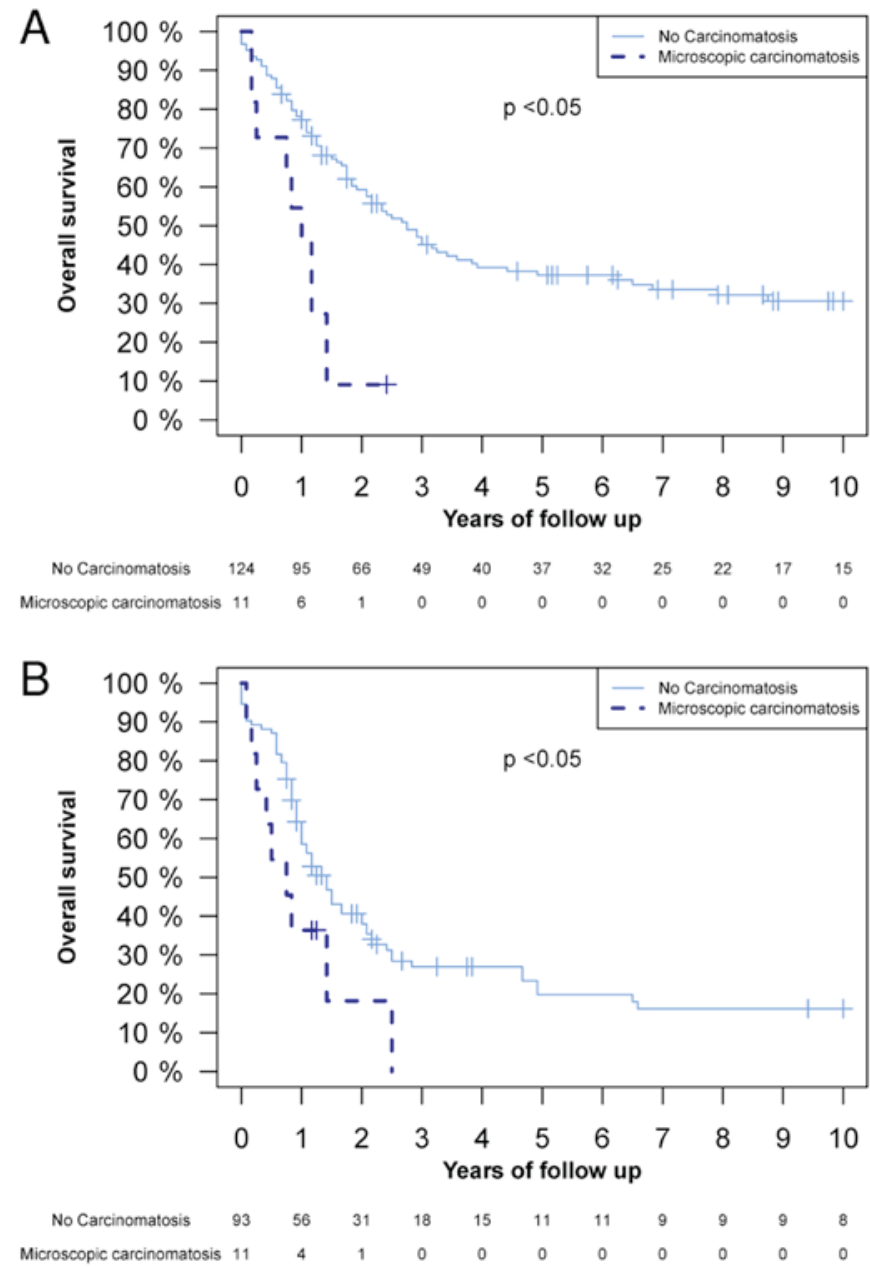

Figure 2. Kaplan-Meier overall survival of the following groups, subdivided by the presence or absence of microscopic peritoneal carcinomatosis: (A) TNM stage II; and (B) TNM stage III. P-values were calculated using a log-rank test.

OS rate between patients who exhibited microscopic PC and those who did not were observed, and this difference remained significant subsequent to multivariate analyses and stratification for TNM stage and tumour extent.

Other limitations may include the selection bias and the limited amount of peritoneal tissue examined (greater and lesser omenta and the transverse mesocolon). In particular, the survival data may be affected by only taking into consideration patients without macroscopic PC and those treated surgically with curative intent. The analyses of the present study was intended to assess whether or not there was a clinical basis for the application of advanced loco-regional cancer treatments, including HIPEC or early postoperative intraperitoneal chemotherapy, for patients with an absence of macroscopic PC who were treated with radical surgery with curative intent. Therefore, the aim of the present study was to determine the role of microscopic PC not identified by clinical examination at the time of surgery. The present study included only patients who received standard surgical treatment for gastric cancer, excluding cases affected by macroscopic PC and those treated with peritonectomy and HIPEC, and the location of excised peritoneum included only greater omenta, lesser omenta and transverse mesocolon. In particular, the prevalence of microscopic PC observed in the study population (5.5\%) was higher compared with that in a similar, previously published study (2.7\%) (13). Liu et al (13) considered only lesser omenta, greater omenta and transverse mesocolon locations, however, they did not select their population and included patients receiving palliative surgery in the prevalence denominator. Therefore, it is expected that in the present study the prevalence of microscopic PC would be higher compared with that presented by Liu et al (13), as the present study only included patients without evident PC at the time of surgery. In this previous study (13) and the present study, there is a potential underestimation of the real prevalence of microscopic PC due to the limited peritoneal tissue examined. These prevalence values of the previous study (13) and the present study appear lower compared with those calculated using positive peritoneal fluid cytology (14).

Among the patients treated with radical surgical resection, the most frequent recurrence site of gastric cancer is the peritoneum (15), which also represents the most common site of metastasis in patients with stage IV gastric cancer, and one of the leading causes of patient mortality $(16,17)$. In addition, the occasional identification of isolated small peritoneal metastasis appears to not be considered in the classification of PC in gastric cancer (15). In a previously published article about patients with microscopic PC, a shorter survival time was demonstrated compared with patients without microscopic PC, but a longer survival rate was identified compared with patients with macroscopic PC (15). In accordance with this study, the present study observed a significantly shorter OS rate among patients affected by microscopic PC compared with patients without microscopic PC.

A previous study described the use of peritoneal fluid cytology as a prognostic factor for gastric cancer (18). The presence of intraperitoneal free cancer cells appears to increase peritoneal recurrence risk, and to predict poorer OS in patients affected by gastric cancer (18). However, the role that the presence or the absence of intraperitoneal free cancer cells should have in the management of gastric cancer remains unclear, as there is no uniform way of interpreting the results of peritoneal fluid cytology analyses (18). The presence of intraperitoneal free cancer cells may be a sign of microscopic PC that intra-operative examination and routine histology fail to detect; however, this requires further investigation.

Knowing the high likelihood of the presence of microscopic $\mathrm{PC}$ at the time of primary surgery for gastric cancer through the identification of predictive factors or by novel rapid diagnostic techniques will improve patient survival, through enabling the selection of more aggressive treatments for these patients, including HIPEC or emerging novel advanced loco-regional treatment options like intraperitoneal immunotherapy (19). The present study revealed that adenocarcinoma a diffuse type, lymphatic and vascular invasion, cancer location at the site of a previous gastric surgery and a tumour extent $>\mathrm{T} 2$ were significant predictive factors for the presence of microscopic PC in a multivariate model.

In conclusion, the results of the present study indicate that $\geq 5.5 \%$ of patients treated with surgery with a curative intent may benefit from more aggressive loco-regional treatment against microscopic PC at the time of primary surgery. In 
particular, improvements to the efforts made in developing techniques for identifying high-risk patients with microscopic $\mathrm{PC}$ and to administer more aggressive and effective treatments for this group are required.

\section{References}

1. Botterweck AA, Schouten LJ, Volovics A, Dorant E and van Den Brandt PA: Trends in incidence of adenocarcinoma of the oesophagus and gastric cardia in ten European countries. Int J Epidemiol 29: 645-654, 2000.

2. Parkin DM, Bray F, Ferlay J and Pisani P: Global cancer statistics, 2002. CA Cancer J Clin 55: 74-108, 2005.

3. Birri S, Bidoli E, Zucchetto A, Dal Maso L, Zanier L and Serraino D: The tumors in friuli venezia giulia. Data on incidence, survival and prevalence: Update to 2007. Technical report, Regione Autonoma Friuli Venezia Giulia-Regional Epidemiology Service. 2011.

4. D'Angelica M, Gonen M, Brennan MF, Turnbull AD, Bains M and Karpeh MS: Patterns of initial recurrence in completely resected gastric adenocarcinoma. Ann Surg 240: 808-816, 2004.

5. Sadeghi B, Arvieux C, Glehen O, Beaujard AC, Rivoire M, Baulieux J, Fontaumard E, Brachet A, Caillot JL, Faure JL, et al: Peritoneal carcinomatosis from non-gynecologic malignancies: Results of the EVOCAPE 1 multicentric prospective study. Cancer 88: 358-363, 2000.

6. Yang XJ, Huang CQ, Suo T, Mei LJ, Yang GL, Cheng FL, Zhou YF, Xiong B, Yonemura Y and Li Y: Cytoreductive surgery and hyperthermic intraperitoneal chemotherapy improves survival of patients with peritoneal carcinomatosis from gastric cancer: Final results of a phase III randomized clinical trial. Ann Surg Oncol 18 $1575-1581,2011$

7. Pasqual EM, Bertozzi S, Bacchetti S, Londero AP, Basso SM, Santeufemia DA, Lo Re G and Lumachi F: Preoperative assessment of peritoneal carcinomatosis in patients undergoing hyperthermic intraperitoneal chemotherapy following cytoreductive surgery. Anticancer Res 34: 2363-2368, 2014.

8. Pasqual E, Bertozzi S, Bacchetti S and Londero A: Effective therapy in peritoneal neoplasia with low peritoneal cancer index values: The difficulty of diagnosis. Int Onkologie 4: 26-29, 2012
9. Lauren P: The two histological main types of gastric carcinoma: Diffuse and so-called intestinal-type carcinoma. An attempt at a histo-clinical classification. Acta Pathol Microbiol Scand 64: 31-49, 1965

10. Bosman FT, Carneiro F, Hruban RH and Theise ND (eds): World Health Organization Classification of Tumours of the Digestive System. Vol. 3. 4th edition. IARC Press, Lyon, 2010.

11. Japanese Gastric Cancer Association: Japanese classification of gastric carcinoma- 2nd english edition -. Gastric Cancer 1: 10-24, 1998.

12. NationalComprehensiveCancer Network: Gastric Cancer (Version 1.2014). http://www.nccn.org/professionals/physician_gls/pdf/gastric.pdf. Accessed August 29, 2014.

13. Liu X, Cai H, Sheng W and Wang Y: Long-term results and prognostic factors of gastric cancer patients with microscopic peritoneal carcinomatosis. PLoS One 7: e37284, 2012.

14. Oh CA, Bae JM, Oh SJ, Choi MG, Noh JH, Sohn TS and Kim S: Long-term results and prognostic factors of gastric cancer patients with only positive peritoneal lavage cytology. J Surg Oncol 105: 393-399, 2012.

15. Li JH, Zhang SW, Liu J, Shao MZ and Chen L: Review of clinical investigation on recurrence of gastric cancer following curative resection. Chin Med J (Engl) 125: 1479-1495, 2012.

16. Saito H, Tsujitani S, Kondo A, Ikeguchi M, Maeta M and Kaibara N: Expression of vascular endothelial growth factor correlates with hematogenous recurrence in gastric carcinoma. Surgery 125: 195-201, 1999.

17. Sarela AI, Miner TJ, Karpeh MS, Coit DG, Jaques DP and Brennan MF: Clinical outcomes with laparoscopic stage M1, unresected gastric adenocarcinoma. Ann Surg 243: 189-195, 2006.

18. Leake PA, Cardoso R, Seevaratnam R, Lourenco L, Helyer L, Mahar A, Rowsell C and Coburn NG: A systematic review of the accuracy and utility of peritoneal cytology in patients with gastric cancer. Gastric Cancer 1 (Suppl 15): S27-S37, 2012.

19. Goéré D, Gras-Chaput N, Aupérin A, Flament C, Mariette C, Glehen O, Zitvogel L and Elias D: Treatment of gastric peritoneal carcinomatosis by combining complete surgical resection of lesions and intraperitoneal immunotherapy using catumaxomab. BMC Cancer 14: 148, 2014

This work is licensed under a Creative Commons Attribution-NonCommercial-NoDerivatives 4.0 International (CC BY-NC-ND 4.0) License. 